\title{
Translation of waves along quantum vortex filaments in the low-temperature two-dimensional local induction approximation
}

\author{
Robert A. Van Gorder* \\ Mathematical Institute, University of Oxford \\ Andrew Wiles Building, Radcliffe Observatory Quarter, Woodstock Road, Oxford, OX2 6GG, United Kingdom \\ Email: Robert.VanGorder@maths.ox.ac.uk
}

July 28,2015

\begin{abstract}
In a recent paper, we give a study of the purely rotational motion of general stationary states in the two-dimensional local induction approximation (2D-LIA) governing superfluid turbulence in the low-temperature limit [Svistunov, Phys. Rev. B 52 (1995) 3647]. Such results demonstrated that variety of stationary configurations are possible from vortex filaments exhibiting purely rotational motion in addition to commonly discussed configurations such as helical or planar states. However, the filaments (or, more properly, waves along these filaments) can also exhibit translational motion along the axis of orientation. In contrast to the study on vortex configurations for purely rotational stationary states, the present paper considers non-stationary states which exhibit a combination of rotation and translational motion. These solutions can essentially be described as waves or disturbances which ride along straight vortex filament lines. As expected from our previous work, there are a number of types of structures that can be obtained under the 2D-LIA. We focus on non-stationary states, as stationary states exhibiting translation will essentially take the form of solutions studied in [Van Gorder, Phys. Fluids 26 (2014) 065105], with the difference being translation along the reference axis, so that qualitative appearance of the solution geometry will be the same (even if there are quantitative differences). We discuss a wide variety of general properties of these non-stationary solutions, and derive cases in which they reduce to known stationary states. We obtain various routes to Kelvin waves along vortex filaments, and demonstrate that if the phase and amplitude of a disturbance both propagate with the same wave speed, then Kelvin waves will result. We also consider the self-similar solutions to the model, and demonstrate that these types of solutions can model vortex kinks that gradually smooth and radiate Kelvin waves as time increases. Such solutions qualitatively agree with what one might expect from post-reconnection events.
\end{abstract}

Keywords: Vortex dynamics; vortex filament; superfluid turbulence; integrable models

\section{Introduction}

A vortex in the reference frame moving with the superfluid was theoretically studied some time ago $[1,2,3]$. For a quantized vortex filament, the dynamics are taken to be those given by the Biot-Savart law, which is often approximated by a local induction approximation (LIA). Schwarz [4] obtained a type of quantum LIA which takes mutual friction and interaction with the normal 
fluid into account. However, in the very low temperature case, the mutual friction effects are negligible and the vortex dynamics decouple from the normal fluid flow. In the limit where these mutual friction effects are zero, the dynamics of the quantized vortex filament are again given by the Biot-Savart formulation, and approximated by the LIA. The standard Biot-Savart law is

$$
\frac{d \mathbf{r}}{d t}=\frac{\Gamma}{4 \pi} \int \frac{\left(\mathbf{r}_{0}-\mathbf{r}\right) \times d \mathbf{r}_{0}}{\left|\mathbf{r}_{0}-\mathbf{r}\right|^{3}}
$$

while the LIA is $[5,6]$

$$
\mathbf{v}=\gamma \kappa \mathbf{t} \times \mathbf{n},
$$

where $\mathbf{t}$ and $\mathbf{n}$ are unit tangent and unit normal vectors to the vortex filament, respectively, $\kappa$ is the local curvature and $\Gamma$ is the quantized circulation of the filament. The parameter $\gamma=\frac{\Gamma}{2 \pi} \ln \left(\ell / \epsilon_{0}\right)$, where $\epsilon_{0}$ is the vortex core radius and $\ell$ is the characteristic length scale (to remove units from $\epsilon_{0}$ ).

Hasimoto [7] obtained a 1-soliton solution of the LIA in the curvature-torsion frame. Exact stationary solutions to the LIA in extrinsic coordinate space have been discussed by Kida [8] in the case of torus knots, planar solutions, and helices; some of these solutions are given by elliptic integrals. Hasimoto [9] considered a planar vortex filament in the curvature-torsion frame of reference. This influential and often cited paper demonstrates the relation between the curvature of a vortex filament and elastica. Such a solution was also considered by Kida [8], who obtained results in terms of elliptic integrals in the moving (time-dependent) arc length coordinate frame, with stability results for some filaments in this framework later provided [10]. Fukumoto [11] considered the influence of background flows on such stationary states. For the Cartesian frame, some preliminary results were determined in Van Gorder, though only some special solutions were given. There is an alternate formulation, given by Umeki $[12,13]$, which provides the LIA in an arc-length coordinate frame. The Hasimoto filament can be determined exactly in this frame (as is also true of the curvature-torsion frame), and the results were worked out by Van Gorder [14]. Small amplitude space-periodic solutions of planar type were obtained through a multiple scales analysis [15]. Such solutions are valid in the small-amplitude regime when the nonlinearity becomes sufficiently weak, though solutions break down after that.

There is a 2D-LIA which, under certain assumptions on the vortex filament (which we shall outline below), permits us to directly obtain a vortex filament in Cartesian coordinates. In the context of superfluids in the low-temperature limit (i.e., in the absence of superfluid friction parameters), the Cartesian form of the LIA was previously derived by Svistunov [16], where the Cartesian representation of the LIA dynamics are represented as a Hamiltonian system for a single periodic vortex line along one axis. Introducing potential $\Psi(x, t)=Y(x, t)+i Z(x, t)$, Svistunov showed that the Biot-Savart law (1) could be written in Hamiltonian form

$$
i \Psi_{t}=\frac{\delta}{\delta \Psi^{*}} H[\Psi]
$$

where

$$
H[\Psi]=\frac{\Gamma}{4 \pi} \iint \frac{1+\operatorname{Re}\left[\Psi_{x}^{*}\left(x_{1}\right) \Psi_{x}\left(x_{2}\right)\right]}{\sqrt{\left(x_{1}-x_{2}\right)^{2}+\left|\Psi\left(x_{1}\right)-\Psi\left(x_{2}\right)\right|^{2}}} d x_{1} d x_{2} .
$$

This is a type of $2 \mathrm{D}$ Biot-Savart law. Assuming the function $\Psi$ is of sufficient bounded variation (so that it makes physical sense in the context of the vortex filament representation), one may introduce a cutoff at the vortex filament radius $a<\left|\mathbf{r}-\mathbf{r}_{0}\right|$ in order to derive a two-dimensional form of the 
LIA (2D-LIA). In particular, by requiring the filament curve to be of sufficient bounded variation, we mean that $\left|\Psi\left(x_{1}\right)-\Psi\left(x_{2}\right)\right|$ is much smaller than $\left|x_{1}-x_{2}\right|$. Assuming a differentiable filament curve, this is equivalent to $\left|\Psi^{\prime}(x)\right|$ being sufficiently small for all $x$. Therefore, the approximation is valid under a type of double limit in which the cut-off parameter is small and the spatial variation in the curve is also small. One way to ensure the latter is to consider small amplitude solutions. However, it is clear that a line filament, corresponding to any constant $\Psi(x)=\Psi_{0} x$, is a solution no matter how large the modulus $\left|\Psi_{0}\right|$ is taken to be. Therefore, it is more fundamental to view the boundedness condition as one on the variation in the curve, rather than on the particular size of the function $\Psi(x)$, alone. However, in many cases, the latter can imply the former.

Considering this double limit, the Hamiltonian reduces to the 2D-LIA

$$
\hat{H}[\Psi]=2 \frac{\Gamma}{4 \pi} \ln (\ell / a) \int \sqrt{1+\left|\Psi_{x}\left(x_{1}\right)\right|^{2}} d x_{1}=\gamma L[w],
$$

where $\gamma=\frac{\Gamma}{2 \pi} \ln \left(\ell / \epsilon_{0}\right)$ is the filament strength and $L[w]$ is the total vortex line length. So, under the LIA, the Hamiltonian is just a scaling of the total vortex length. In equation (4) of Boffetta et al. [17], it was shown that the equation of motion for this Hamiltonian becomes

$$
i \Psi_{t}+\left(\frac{\Psi_{x}}{\sqrt{1+\left|\Psi_{x}\right|^{2}}}\right)_{x}=0,
$$

where here we have used a different scaling of $t$ to remove a factor of two from (6). It shall be this equation that we are interested in. Symmetry properties of this model have been discussed by Sonin [18]. Helical waves from the Cartesian form of the LIA (6) were considered by Sonin [19]. There are a number of known specific solutions forms to the LIA in both classical and quantum settings. Exact solution forms consist of closed vortex rings and knots [5, 20, 21, 22, 23], or loops due to self-intersection $[4,16,15]$ and the various open filament solutions which consist of helical [19] and planar filaments $[9,8,14,15]$. Another type of filament solution would be the self-similar solutions of the LIA $[24,25,26]$. Solitons have also been found on vortex filaments $[7,13,27]$.

Equation (6) is equivalent to

$$
i \Psi_{t}+\gamma \frac{\Psi_{x x}}{\left(1+\left|\Psi_{x}\right|^{2}\right)^{3 / 2}}+\frac{\gamma}{2} \frac{\Psi_{x}\left(\Psi_{x}^{*} \Psi_{x x}-\Psi_{x} \Psi_{x x}^{*}\right)}{\left(1+\left|\Psi_{x}\right|^{2}\right)^{3 / 2}}=0 .
$$

Note that when $\Psi_{x}^{*} \Psi_{x x}-\Psi_{x} \Psi_{x x}^{*} \rightarrow 0$, we would have no translation of the vortex filaments, as was discussed in previous work, so the filaments would essentially be spatial structures that rotate in time. In [28], solutions with zero translation and pure rotation were considered. Such purelyrotating solutions take the form $\Psi(x, t)=e^{-\gamma i t} \psi(x)$, while the zero-translation condition is given be setting $\Psi_{x}^{*} \Psi_{x x}-\Psi_{x} \Psi_{x x}^{*}=0$ in the governing scalar equation. In the present paper, we are interested in waves that translate along the filament (as opposed to static stationary structures which strictly rotate in time), so we shall need to keep the nonlinear term arising due to translation. Furthermore, since translating stationary states appear geometrically similar to purely rotational stationary states (with specific quantitative differences arising from the extra terms), we shall primarily be concerned with non-stationary solutions. Mathematically, this means that the solutions $\Psi$ will not always have time-independent modulus (as they did in [28]). The need to consider translating waves was discussed in the correspondence $[29,30]$. 
In the present paper we study the translation of waves along vortex filaments under the 2DLIA model (6), and as such these results are complementary to those of [28] (in which purely rotational motion of vortex filaments was studied). In Section 2, we consider more general case where solutions are not assumed to be purely rotational, including a fairly broad class of solutions which is shown to preserve wave action. We also address several specific limits, including the case where phase is constant in space, the case where the amplitude is constant. We study general steady state solutions which are not purely rotational, as well, and we are able to outline the behavior of small-amplitude stationary states under translational motion. In Section 3, we turn out attention to the pure traveling wave solution (in which both the phase and amplitude terms propagate with a constant wave speed along the filament). We prove that when both phase and amplitude propagate at the same wave speed, the result corresponds to Kelvin waves along a shifted line vortex filament. In Section 4 we obtain similarity solutions to the model (6), and we demonstrate how these solutions share qualitative features of vortex kinks and post-reconnection events. While extremely sharp kinks are forbidden by LIA (in such a case, one would want to simulate the full Biot-Savart dynamics), we are able to demonstrate a class of solution with kinks that gradually dissipate in time, and we can prove that such solutions stay within the bounds of what one may consider under the 2D-LIA. We finally provide a discussion of the results in Section 5 , relating some of the obtained solutions back to numerical or experimental results present in the literature.

\section{General first integral and solution properties}

Now that we have considered stationary states (in the previous section, and elsewhere [28]), we shall next turn our attention to non-stationary solutions. In this section, we shall consider general properties of the vortex filament solutions to the 2D-LIA. We first obtain a first integral for the 2D-LIA, and use this to comment on the wide class of wave action preserving solutions possible. We shall determine when general features of either the phase or amplitude imply that the solutions to (6) can be completely classified, showing that the only solutions with constant phase in space is the planar filament, and the only constant amplitude solution corresponds to a helical filament. We also consider various limiting behaviors.

\subsection{A type of first integral for the 2D-LIA model}

Let us obtain a type of first integral for the LIA reduction of the Svistunov equation, (6). First, take the complex conjugate of (6) and multiply by $\Psi$, then multiply (6) by the complex conjugate $\Psi^{*}$, and finally subtract one from the other to obtain

$$
i \frac{\partial}{\partial t}|\Psi|^{2}+\Psi^{*}\left(\frac{\Psi_{x}}{\sqrt{1+\left|\Psi_{x}\right|^{2}}}\right)_{x}-\Psi\left(\frac{\Psi_{x}^{*}}{\sqrt{1+\left|\Psi_{x}\right|^{2}}}\right)_{x}=0 .
$$

Integrating over the entire spatial domain, we have

$$
i \frac{\partial}{\partial t} \int_{-\infty}^{\infty}|\Psi|^{2} d x+\int_{-\infty}^{\infty}\left\{\Psi^{*}\left(\frac{\Psi_{x}}{\sqrt{1+\left|\Psi_{x}\right|^{2}}}\right)_{x}-\Psi\left(\frac{\Psi_{x}^{*}}{\sqrt{1+\left|\Psi_{x}\right|^{2}}}\right)_{x}\right\} d x=0
$$


or, performing the latter integration,

$$
\frac{\partial}{\partial t} \int_{-\infty}^{\infty}|\Psi|^{2} d x+\left.\frac{\Psi^{*} \Psi_{x}-\Psi \Psi_{x}^{*}}{\sqrt{1+\left|\Psi_{x}\right|^{2}}}\right|_{x \rightarrow-\infty} ^{x \rightarrow+\infty}=0
$$

Write $\Psi(x, t)$ in terms of the amplitude and phase, $\Psi(x, t)=R(x, t) \exp (i \Theta(x, t))$. Also note that the integral over the modulus squared gives us the wave action of the vortex filament, $E$, over the real space. $E$ is not generally constant, but rather depends on time, so that $E=E(t)$. From the above derivation, we see that this wave action depends strictly on the asymptotic spatial behavior of the phase and amplitude of the potential function $\Psi$ like

$$
\frac{d E}{d t}=-\left.\frac{2 R^{2} \Theta_{x}}{\sqrt{1+R_{x}^{2}+R^{2} \Theta_{x}^{2}}}\right|_{x \rightarrow-\infty} ^{x \rightarrow+\infty} .
$$

There are a number of ways we might obtain a wave action preserving solution. First, if $\Theta_{x} \rightarrow 0$ as $x \rightarrow \pm \infty$, then $E(t)=E_{0}$, a constant, hence wave action is preserved. If $\Psi$ and $\Psi_{x}$ are symmetric over space (that is, $\Psi(-x, t)=\Psi(x, t)$ and $\Psi_{x}(-x, t)=\Psi_{x}(x, t)$ ), then $E(t)=E_{0}$. If the modulus $R$ of a solution decays sufficiently rapidly as $x \rightarrow \pm \infty$, then $E(t)=E_{0}$.

For example, the planar filament solution satisfies $\Theta_{x}=0$ for all $x$, so the planar vortex filament preserves wave action over time. For the helical filament, the quantity

$$
\frac{2 R^{2} \Theta_{x}}{\sqrt{1+R_{x}^{2}+R^{2} \Theta_{x}^{2}}}=\frac{2 A^{2} k}{\sqrt{1+A^{2} k^{2}}}
$$

is a constant, hence after evaluating at both asymptotic limits we have zero. As such, the helical filaments also preserve wave action.

\subsection{General small-amplitude stationary states}

The most general stationary states can be found by taking a solution of the form

$$
\Psi(x, t)=R(x) \exp \left(i\left[\Theta(x)-\omega t+x_{0}\right]\right),
$$

where $\omega$ is the spectral parameter, $x_{0}$ is a constant, and $R$ and $\Theta$ are real-valued functions of the space variable. Including both rotational and translational terms (note only rotational terms were kept in [28], for reasons discussed above) we have

$$
\omega R e^{i \Theta}+\left(\frac{\left(R^{\prime}+i R \Theta^{\prime}\right) e^{i \Theta}}{\sqrt{1+R^{\prime 2}+R^{2} \Theta^{\prime 2}}}\right)_{x}=0 .
$$

Separating this equation into real and imaginary parts, we obtain the coupled system

$$
\begin{gathered}
\omega R-\frac{R \Theta^{\prime 2}}{\sqrt{1+R^{\prime 2}+R^{2} \Theta^{\prime 2}}}+\left(\frac{R^{\prime}}{\sqrt{1+R^{\prime 2}+R^{2} \Theta^{\prime 2}}}\right)^{\prime}=0, \\
\frac{R^{\prime} \Theta^{\prime}}{\sqrt{1+{R^{\prime}}^{2}+R^{2} \Theta^{\prime 2}}}+\left(\frac{R \Theta^{\prime}}{\sqrt{1+R^{\prime 2}+R^{2} \Theta^{\prime 2}}}\right)^{\prime}=0
\end{gathered}
$$


which is again more complicated than that of [28], since translational motion along the filament length is included. The solutions mentioned previously in this section are of course special case solutions of these equations, while the solutions of [28] are special cases if only the rotational part of these equations is maintained.

Equations (15)-(16) are too complicated to admit closed-form analytical solutions, and therefore must be integrated numerically. One obtains a variety of solutions in this manner, with qualitative features similar to those of [28], so we shall not duplicate those qualitative results here. In the small amplitude limit, let us assume $R(x)=\epsilon \hat{R}(x)$ where $0<\epsilon<<1$ is a small parameter and $\hat{R}(x)$ is a function of order unity. Equations (15)-(16) then scale as

$$
\begin{gathered}
\omega \hat{R}-\hat{R} \Theta^{\prime 2}+\hat{R}^{\prime \prime}=O\left(\epsilon^{2}\right), \\
2 \hat{R}^{\prime} \Theta^{\prime}+\hat{R} \Theta^{\prime \prime}=O\left(\epsilon^{2}\right) .
\end{gathered}
$$

Assuming a positive solution $\hat{R}(x)>0$ (we prove this assumption is valid later), the second of these equations gives a solution of the form

$$
\Theta(x) \approx \Theta_{0} \int_{0}^{x} \frac{d \sigma}{(\hat{R}(\sigma))^{2}}+\Theta_{1},
$$

and we can set $\Theta_{1}=0$ since $x_{0}$ already holds an arbitrary constant phase shift. This then puts the first equation into the form

$$
\omega \hat{R}-\frac{\Theta_{0}}{\hat{R}}+\hat{R}^{\prime \prime}=0 .
$$

Multiplying by $2 \hat{R}^{\prime}$ and integrating, we obtain the first integral

$$
\omega \hat{R}^{2}-2 \Theta_{0} \ln (\hat{R})+\left(\hat{R}^{\prime}\right)^{2}=I
$$

where $I$ is a constant of integration. Since we seek a bounded solution $\hat{R}(x)$ of order unity, let us give the conditions $\hat{R}(0)=1$ and $\hat{R}^{\prime}(0)=0$. This gives $I=\omega$. So, in the small amplitude limit, stationary states should obey the implicit relation

$$
\pm x=\int_{1}^{\hat{R}(x)} \frac{d \nu}{\sqrt{\omega\left(1-\nu^{2}\right)+2 \Theta_{0} \ln (\nu)}} .
$$

From the first integral, we must have

$$
\omega\left(1-\hat{R}^{2}\right)+2 \Theta_{0} \ln (\hat{R}) \geq 0 .
$$

Asymptotically, the term on the left hand side is negative as $\hat{R} \rightarrow 0$ and as $\hat{R} \rightarrow \infty$. Therefore, there must exist a finite region $0<\hat{R}<\infty$ for which the condition (23) holds. As a function of $\hat{R}$, the term on the left hand side of (23) has one or two roots, depending on the relative values of $\omega$ and $\Theta_{0}$. Note that $\hat{R}=1$ is always a root. We summarize the possibilities as follows.

- If $0<\frac{\Theta_{0}}{\omega}<1$ and $\omega>0$, then there exists a root $0<\hat{R}_{-}<1$ to $\omega\left(1-\hat{R}^{2}\right)+2 \Theta_{0} \ln (\hat{R})$ such that $\hat{R}_{-} \leq \hat{R}(x) \leq 1$ for all $x \in \mathbb{R}$.

- If $\frac{\Theta_{0}}{\omega}>1$ and $\omega>0$, then there exists a root $\hat{R}_{+}>1$ to $\omega\left(1-\hat{R}^{2}\right)+2 \Theta_{0} \ln (\hat{R})$ such that $1 \leq \hat{R}(x) \leq \hat{R}_{+}$for all $x \in \mathbb{R}$. 
- If $\frac{\Theta_{0}}{\omega}=1$ and $\omega>0$, then there exists only the constant solution $\hat{R}(x) \equiv 1$ for all $x \in \mathbb{R}$.

- If one or both of $\omega$ and $\Theta_{0}$ are not positive, then there is no strictly positive solution $\hat{R}(x)$.

Therefore, when both $\omega$ and $\Theta_{0}$ are positive, there exists a small curvature generalized stationary solution in which both curvature and torsion depend on the spatial domain. Such solutions take the form

$$
\Psi(x, t)=\epsilon \hat{R}(x) \exp \left(i\left\{\Theta_{0} \int_{0}^{x} \frac{d \sigma}{(\hat{R}(\sigma))^{2}}-\omega t+x_{0}\right\}\right),
$$

where $\hat{R}(x)$ is a strictly positive periodic function. If $\hat{R}(x)$ is taken as a constant, (24) gives a helix (such solutions were previously studied [19]), while if $\Theta_{0}=0$, then (24) describes a planar filament (such solutions were previously studied $[9,8,14,15]$ ). More generally, this solution should exhibit some type of coiled structure, although it need not be as regular as a helix. For the intermediate cases, we will see filament solutions similar to those observed in [28], with the difference being that the present small amplitude solutions take into account translation along the reference axis, as opposed to just rotational dynamics.

\section{3 $\Theta_{x}=0$ implies planar filament}

From above, we see that there are multiple scenarios in which the total wave action of the vortex filament might be conserved in time. One possibility is to have the phase satisfy $\Theta_{x}=0$. That is, the phase is independent of the spatial coordinate. Such a solution then takes the form $\Psi(x, t)=R(x, t) \exp (i \Theta(t))$. Since both $R$ and $\Theta$ are real-valued functions, placing such a solution representation into (6), we obtain $R_{t}=0$ and $R \Theta_{t}=\left(R_{x}\left[1+R_{x}^{2}\right]^{-1 / 2}\right)_{x}$. Since $R_{t}=0$, we must have that $R$ is independent of $t$, hence $R=R(x)$. Then, the right hand side of the second differential equation is a strict function of $x$, meaning that $\Theta_{t}$ must take the form of a constant. As such, we must have $\Theta(t)=-\omega_{1} t+\omega_{0}$, for real constants $\omega_{1}$ and $\omega_{0}$. With this, the second equation becomes $\omega_{1} R+\left[1+R_{x}^{2}\right]^{-3 / 2} R_{x x}=0$, which is exactly the planar filament equation. Therefore, when the phase of a solution $\Psi$ to the 2D-LIA (6) is constant in space, the only possible solution is a planar solution.

\section{4 $R=$ constant implies helical filament}

Let us now assume that a solution to (6) takes the form $\Psi(x, t)=R_{0} \exp (i \Theta(x, t))$. Placing this solution into (6), and separating real and imaginary parts of the resulting equation, we obtain

$$
\Theta_{t}+\frac{\Theta_{x}^{2}}{\sqrt{1+R_{0}^{2} \Theta_{x}^{2}}}=0 \text { and }\left(\frac{\Theta_{x}}{\sqrt{1+R_{0}^{2} \Theta_{x}^{2}}}\right)_{x}=0 .
$$

From the second equation, we must have $\Theta(x, t)=K_{1}(t) x+K_{2}(t)$ for some functions $K_{1}$ and $K_{2}$ which depend on $t$ alone. Placing this representation into the first equation,

$$
K_{1}^{\prime}(t) x+K_{2}^{\prime}(t)+\frac{K_{1}(t)^{2}}{\sqrt{1+R_{0}^{2} K_{1}(t)^{2}}}=0,
$$

which implies $K_{1}^{\prime}(t)=0$ and hence $K_{1}(t)=k$, a constant. Then, $K_{2}(t)=-k^{2}\left[1+R_{0}^{2} k^{2}\right]^{-1 / 2} t$. As such, any constant modulus solution must necessarily take the form of a helix. 


\section{Pure traveling wave solutions for $\Psi$}

Here we show that the only purely traveling wave solutions to the Svistunov model are helix-like with both the phase and amplitude propagate in the same manner. We assume that the function $\Psi(x, t)$ depends on a single wave variable, $z=x-c t$ where $c$ is the wave speed. Both the phase and modulus of the solution depend on the same wave variable, and therefore propagation of each is at rate $c$. For this reason, we say that $\Psi$ is a pure traveling wave solution, in contrast to the case where the phase and amplitude of $\Psi$ are each wavelike but with different propagation constants. Then, we may write

$$
\Psi(x, t)=\psi(z)=\rho(z) \exp (i \phi(z)) \quad \text { with } \quad z=x-c t,
$$

which puts (6) into the form

$$
-c i \psi^{\prime}+\left(\frac{\psi^{\prime}}{\sqrt{1+\left|\psi^{\prime}\right|^{2}}}\right)^{\prime}=0,
$$

where prime denotes differentiation with respect to the wave variable $z$. This equation admits the exact first integral

$$
-c i \psi+\frac{\psi^{\prime}}{\sqrt{1+\left|\psi^{\prime}\right|^{2}}}=I_{0},
$$

which in polar coordinates gives the system

$$
\frac{\rho^{\prime}+i \rho \phi^{\prime}}{\sqrt{1+\rho^{\prime 2}+\rho^{2} \phi^{\prime 2}}}=I_{0} e^{-i \phi}+c i \rho .
$$

Here, $I_{0}$ is a constant. If $I_{0}=0$, then $\rho=\rho_{0}$ is constant, while $\phi(z)=\alpha z$ for some constant $\alpha$. This is a helical filament (corresponding to a plane wave solution $\Psi$, as discussed in Section 3).

Let us assume $I_{0} \neq 0$, and write $I_{0}=I_{1}+i I_{2}$. Then, we obtain the real system

$$
\begin{aligned}
& \frac{\rho^{\prime}}{\sqrt{1+\rho^{\prime 2}+\rho^{2} \phi^{\prime 2}}}=I_{1} \cos (\phi)+I_{2} \sin (\phi), \\
& \frac{\rho \phi^{\prime}}{\sqrt{1+{\rho^{\prime}}^{2}+\rho^{2} \phi^{\prime 2}}}=c \rho-I_{1} \sin (\phi)+I_{2} \cos (\phi) .
\end{aligned}
$$

We can rearrange this system to obtain explicit expressions for the derivative terms, finding

$$
\begin{aligned}
\rho^{\prime 2} & =\frac{\left(I_{1} \cos (\phi)+I_{2} \sin (\phi)\right)^{2}}{1-I_{1}^{2}-I_{2}^{2}+2 c\left(I_{1} \sin (\phi)-I_{2} \cos (\phi)\right) \rho-c^{2} \rho^{2}}, \\
\rho^{2} \phi^{\prime 2} & =\frac{\left(c \rho-I_{1} \sin (\phi)+I_{2} \cos (\phi)\right)^{2}}{1-I_{1}^{2}-I_{2}^{2}+2 c\left(I_{1} \sin (\phi)-I_{2} \cos (\phi)\right) \rho-c^{2} \rho^{2}} .
\end{aligned}
$$

\subsection{Equilibrium states for the system (32)-(33)}

Assume that an equilibrium solution $\psi^{*}=\rho^{*} \exp \left(i \phi^{*}\right)$ exists for the system (32)-(33). Then, we must have

$$
I_{1} \cos \left(\phi^{*}\right)+I_{2} \sin \left(\phi^{*}\right)=0
$$


and

$$
c \rho^{*}=I_{1} \sin (\phi *)-I_{2} \cos \left(\phi^{*}\right) .
$$

If $I_{1}=0$, then we have $\phi^{*}=0$ and $\rho^{*}=-\frac{I_{2}}{c}$. If $I_{2}=0$, then we have $\phi^{*}= \pm \frac{\pi}{2}$ and $\rho^{*}= \pm \frac{I_{1}}{c}$. If both $I_{1} \neq 0$ and $I_{2} \neq 0$, then (34) implies

$$
\tan \left(\phi^{*}\right)=-\frac{I_{1}}{I_{2}}
$$

hence

$$
\phi^{*}=-\tan ^{-1}\left(\frac{I_{1}}{I_{2}}\right)
$$

which always gives a unique solution $-\frac{\pi}{2}<\phi^{*}<\frac{\pi}{2}$. Using this value of $\phi^{*}$ in (35), we obtain

$$
c \rho^{*}=-I_{1} \sin \left(\tan ^{-1}\left(\frac{I_{1}}{I_{2}}\right)\right)-I_{2} \cos \left(\tan ^{-1}\left(\frac{I_{1}}{I_{2}}\right)\right)=-\operatorname{sgn}\left(I_{2}\right) \sqrt{I_{1}^{2}+I_{2}^{2}} .
$$

Therefore,

$$
\rho^{*}=-\frac{\operatorname{sgn}\left(I_{2}\right)}{c} \sqrt{I_{1}^{2}+I_{2}^{2}}
$$

and we have the equilibrium solution

$$
\psi^{*}=-\frac{\operatorname{sgn}\left(I_{2}\right)}{c} \sqrt{I_{1}^{2}+I_{2}^{2}} \exp \left(-i \tan ^{-1}\left(\frac{I_{1}}{I_{2}}\right)\right) .
$$

\subsection{Any solution of (32)-(33) yields Kelvin waves along a shifted line filament}

We shall show now that the dynamics of the system (32)-(33) are always consistent with a circle parameterized by the wave variable $z$. Consider $\psi(z)=\rho(z) e^{i \phi(z)}=\hat{Y}(z)+i \hat{Z}(z)$. Then, the general equation of a circle in these coordinates is

$$
\left(\rho \cos (\phi)-a_{1}\right)^{2}+\left(\rho \sin (\phi)+a_{2}\right)^{2}=\left(\hat{Y}-a_{1}\right)^{2}+\left(\hat{Z}+a_{2}\right)=r_{0}^{2},
$$

which gives a circle of radius $r_{0}>0$ centered at $(\hat{Y}, \hat{Z})=\left(a_{1},-a_{2}\right)$. Expanding, we get

$$
\rho^{2}-2 \rho\left(a_{1} \cos (\phi)-a_{2} \sin (\phi)\right)=r_{0}^{2}-a_{1}^{2}-a_{2}^{2} .
$$

Differentiation with respect to $z$ yields, after some algebraic manipulations,

$$
\rho^{\prime}\left(\rho-a_{1} \cos (\phi)+a_{2} \sin (\phi)\right)=-\rho \phi^{\prime}\left(a_{1} \sin (\phi)+a_{2} \cos (\phi)\right) .
$$

Squaring both sides, we obtain

$$
\rho^{\prime 2}\left(\rho-a_{1} \cos (\phi)+a_{2} \sin (\phi)\right)^{2}=\left(\rho \phi^{\prime}\right)^{2}\left(a_{1} \sin (\phi)+a_{2} \cos (\phi)\right)^{2} .
$$

At this point, we substitute values of $\rho^{\prime 2}$ and $\left(\rho \phi^{\prime}\right)^{2}$ from the system (32)-(33) into (44), which then takes the form

$$
\left(I_{1} \cos (\phi)+I_{2} \sin (\phi)\right)^{2}\left(\rho-a_{1} \cos (\phi)+a_{2} \sin (\phi)\right)^{2}=\left(c \rho-I_{1} \sin (\phi)+I_{2} \cos (\phi)\right)^{2}\left(a_{1} \sin (\phi)+a_{2} \cos (\phi)\right)^{2} .
$$


This equation is satisfied provided that $a_{1}=-\frac{I_{2}}{c}$ and $a_{2}=-\frac{I_{1}}{c}$. What this tells us is that the solution to the system (32)-(33) falls on a circle centered at $\left(-\frac{I_{2}}{c}, \frac{I_{1}}{c}\right)$. In other words, we may write

$$
\psi(z)=\hat{\psi}(z)+\frac{i}{c}\left(I_{1}+i I_{2}\right)
$$

where $\hat{\psi}(z)$ corresponds to a parameterization of the circle centered at the origin, hence $\hat{\psi}(z)=$ $r_{0} e^{\alpha i z}$. Placing this solution directly into (29), we directly obtain the algebraic condition $c=$ $\alpha\left(1+\alpha^{2} r_{0}^{2}\right)^{-1 / 2}$. This gives

$$
\alpha= \pm \frac{c}{\sqrt{1-c^{2} r_{0}^{2}}}
$$

which restricts the amplitude of the wave like $0 \leq r_{0}<\frac{1}{|c|}$. Therefore,

$$
\Psi(x, t)=\psi(z)=r_{0} \exp \left( \pm \frac{i c z}{\sqrt{1-c^{2} r_{0}^{2}}}\right)+\frac{i I_{1}-I_{2}}{c} .
$$

Converting this into a filament curve, we have obtain

$$
\mathbf{r}(x, t)=\left(x, r_{0} \cos \left(\frac{c(x-c t)}{\sqrt{1-c^{2} r_{0}^{2}}}\right)-\frac{I_{2}}{c}, \pm r_{0} \sin \left(\frac{c(x-c t)}{\sqrt{1-c^{2} r_{0}^{2}}}\right)+\frac{I_{1}}{c}\right) .
$$

This gives a shifted helix. Therefore, we have shown that a pure traveling wave solution should always take the form of Kelvin waves along a shifted vortex filament. As the wave speed increases, the possible maximal amplitude decreases, since we must have $0 \leq r_{0}<\frac{1}{c \mid}$. This makes sense, as the Kelvin waves must tighten in order to hold their form at large propagation velocities.

\subsection{Relation to other types of waves}

There results suggest that traveling wave solutions that propagate with the same rate (wave speed) in both phase and amplitude (which is the case where the function $\Phi$ itself depends on a wave variable alone) should be limited to Kelvin waves. While this is rather restrictive, note that it makes sense in light of the fact that the most frequently observed solutions in experiments are Kelvin waves. The other kinds of solutions observed (planar solutions, self-similar solutions) are stationary or quasi-stationary, with the phase and amplitude behaving in very different ways, and can not be considered traveling waves. Note that in the case where one assumes that the phase and amplitude differ in wave speed, this restrictive finding is not necessarily true. Indeed, soliton solutions may be possible for equation (6) in cases where the phase and amplitude propagate differently from one another, and have been reported in the literature for related models (such as the cubic NLS obtained from the standard LIA). Since the scalar equation (6) considered here is more complicated than the cubic NLS due to a nonlinearity involving the highest space derivative, the method for obtaining soliton solutions via inverse scattering is much more involved, and will be outlined in a separate work in which the integrability of equation (6) will be exploited so that the inverse scattering transform may be used. Such solutions will take the form $\Psi(x, t)=\rho\left(z_{1}\right) \exp \left(i \phi\left(z_{2}\right)\right)$, where $z_{1}=x-c_{1} t$ and $z_{2}=x-c_{2} t$ are wave variables with $c_{1} \neq c_{2}$. 


\section{Similarity solutions to the 2D-LIA model}

We now consider self-similar solutions to the 2d-LIA model. Such solutions were obtained for different yet related models $[24,25,26]$, so one should expect such solutions to exist for the present model. These serve as another example of solutions which have time dependent amplitude and phase. We consider the solution

$$
\Psi(x, t)=\sqrt{2 t} \hat{\Phi}(\eta) \quad \text { where } \quad \eta=\frac{x}{\sqrt{2 t}} .
$$

Such a class of solutions before have been considered numerically and analytically for other models under LIA in the past. However, as we shall show below, these solutions can be described exactly in closed for under the Svistunov model. The transformation (50) puts (6) into the form

$$
i\left(\hat{\Phi}-\eta \hat{\Phi}^{\prime}\right)+\left(\frac{\hat{\Phi}^{\prime}}{\sqrt{1+\left|\hat{\Phi}^{\prime}\right|^{2}}}\right)^{\prime}=0,
$$

where prime denotes differentiation with respect to the similarity variable, $\eta$. There exists a simple solution $\hat{\Phi}(\eta)=m \eta$ for any constant $m \in \mathbb{C}$, and this gives $\Psi(x, t)=m x$ which is simply equivalent to a straight line filament $\mathbf{r}(x, t)=(x, \operatorname{Re}(m) x, \operatorname{Im}(m) x)$. To construct a more interesting class of solutions, we search for complementary solutions which remain a bounded distance away from the reference axis.

\subsection{Approximation of self-similar dynamics}

Assuming a solution which remains a small distance from the reference axis, let us take $\hat{\Phi}(\eta)=$ $\epsilon \Phi(\eta)$, where $\epsilon$ is a small parameter. Then, we have

$$
i\left(\Phi-\eta \Phi^{\prime}\right)+\Phi^{\prime \prime}=O\left(\epsilon^{2}\right) .
$$

One solution is obviously $\Phi_{1}(\eta)=\eta$. To find a second solution, we write $\Phi_{2}(\eta)=\eta v(\eta)$, which gives us the differential equation

$$
\eta v^{\prime \prime}+\left(2-i \eta^{2}\right) v^{\prime}=0 .
$$

Then, a second solution is

$$
v^{\prime}(\eta)=-\frac{1}{\eta^{2}} \exp \left(\frac{i}{2} \eta^{2}\right)=-\frac{1}{\eta^{2}} \cos \left(\frac{1}{2} \eta^{2}\right)-\frac{i}{\eta^{2}} \sin \left(\frac{1}{2} \eta^{2}\right) .
$$

Integrating as needed,

$$
v(\eta)=\frac{1}{\eta} \cos \left(\frac{1}{2} \eta^{2}\right)+\sqrt{\pi} S\left(\frac{\eta}{\sqrt{\pi}}\right)+i\left\{\frac{1}{\eta} \sin \left(\frac{1}{2} \eta^{2}\right)-\sqrt{\pi} C\left(\frac{\eta}{\sqrt{\pi}}\right)\right\},
$$

where $S$ and $C$ are the Fresnel Sine and Cosine integrals. The second solution is then

$$
\Phi_{2}(\eta)=\cos \left(\frac{1}{2} \eta^{2}\right)+\sqrt{\pi} \eta S\left(\frac{\eta}{\sqrt{\pi}}\right)+i\left\{\sin \left(\frac{1}{2} \eta^{2}\right)-\sqrt{\pi} \eta C\left(\frac{\eta}{\sqrt{\pi}}\right)\right\} .
$$


A complete homogeneous solution is then given by

$$
\Phi(\eta)=c_{1} \eta+c_{2} \Phi_{2}(\eta)
$$

for complex-valued $c_{1}$ and $c_{2}$. We need to ensure that the solution remains bounded. Note that each of the solutions scale as linear functions, so $c_{1}$ and $c_{2}$ need to be picked to ensure the desired asymptotic behavior. It will be useful to obtain a solution for $\eta>0$ and $\eta<0$ and then to match the solutions, as each of the $\eta>0$ and $\eta<0$ solutions will hold a different asymptotic behavior. Note that

$$
\lim _{x \rightarrow \pm \infty} S(x)= \pm \frac{1}{2}=\lim _{x \rightarrow \pm \infty} C(x) .
$$

Picking the real and imaginary parts of the constants $c_{1}$ and $c_{2}$ as needed to prevent divergence, and writing the remaining component as $c \in \mathbb{R}$, we have

$$
\begin{aligned}
\frac{\Phi(\eta)}{c}= & \cos \left(\frac{1}{2} \eta^{2}\right)-\sin \left(\frac{1}{2} \eta^{2}\right)+\sqrt{\pi} \eta\left(S\left(\frac{\eta}{\sqrt{\pi}}\right)+C\left(\frac{\eta}{\sqrt{\pi}}\right)-\operatorname{sgn}(\eta)\right) \\
& +i\left\{\cos \left(\frac{1}{2} \eta^{2}\right)+\sin \left(\frac{1}{2} \eta^{2}\right)+\sqrt{\pi} \eta\left(S\left(\frac{\eta}{\sqrt{\pi}}\right)-C\left(\frac{\eta}{\sqrt{\pi}}\right)\right)\right\},
\end{aligned}
$$

where the $\operatorname{sgn}(\eta)= \pm 1$ term is present to give the appropriate asymptotic scaling after matching. Each of the real and imaginary parts of this function have maximal absolute value equal to one, so we can pick the scaling parameter $c=1$. Then, $\Psi(x, t)=\epsilon \sqrt{2 t} \Phi(\eta)+O\left(\epsilon^{3}\right)$, and putting this back into Cartesian coordinates, the vortex filament is given by the curve

$\mathbf{r}(x, t)=\left[\begin{array}{c}x \\ Y(x, t) \\ Z(x, t)\end{array}\right]=\left[\begin{array}{c}x \\ \epsilon \sqrt{2 t}\left(\cos \left(\frac{x^{2}}{4 t}\right)-\sin \left(\frac{x^{2}}{4 t}\right)\right)+\epsilon \sqrt{\pi} x\left(S\left(\frac{x}{\sqrt{2 \pi t}}\right)+C\left(\frac{x}{\sqrt{2 \pi t}}\right)-\operatorname{sgn}(x)\right)+O\left(\epsilon^{3}\right) \\ \epsilon \sqrt{2 t}\left(\cos \left(\frac{x^{2}}{4 t}\right)+\sin \left(\frac{x^{2}}{4 t}\right)\right)+\epsilon \sqrt{\pi} x\left(S\left(\frac{x}{\sqrt{2 \pi t}}\right)-C\left(\frac{x}{\sqrt{2 \pi t}}\right)\right)+O\left(\epsilon^{3}\right)\end{array}\right]$.

We plot the similarity solution in Figure 1. For this solution, we have scaled out the linear trend in the solutions, so as $|x|$ becomes large, the solution tends to a line filament along the $x$-axis. If we were to have included this linear trend, then for large $|x|$, the solution would tend to two separate line filaments for large enough $|x|$. We shall see this latter type of behavior numerically.

\subsection{Numerical simulation of self-similar dynamics}

In order verify the analytical approximation method (valid for small $\epsilon$ ) against numerical solutions, we shall consider (51) into a real system. Writing $\hat{\Phi}(\eta)=p(\eta)+i q(\eta)$, we have

$$
\begin{gathered}
p-\eta p^{\prime}+\left(\frac{q^{\prime}}{\sqrt{1+p^{\prime 2}+{q^{\prime 2}}^{2}}}\right)^{\prime}=0, \\
-q+\eta q^{\prime}+\left(\frac{p^{\prime}}{\sqrt{1+{p^{\prime}}^{2}+{q^{\prime}}^{2}}}\right)^{\prime}=0,
\end{gathered}
$$

with initial conditions

$$
p(0)=p_{0} \quad p^{\prime}(0)=p_{1} \quad q(0)=q_{0} \quad q^{\prime}(0)=q_{1} .
$$




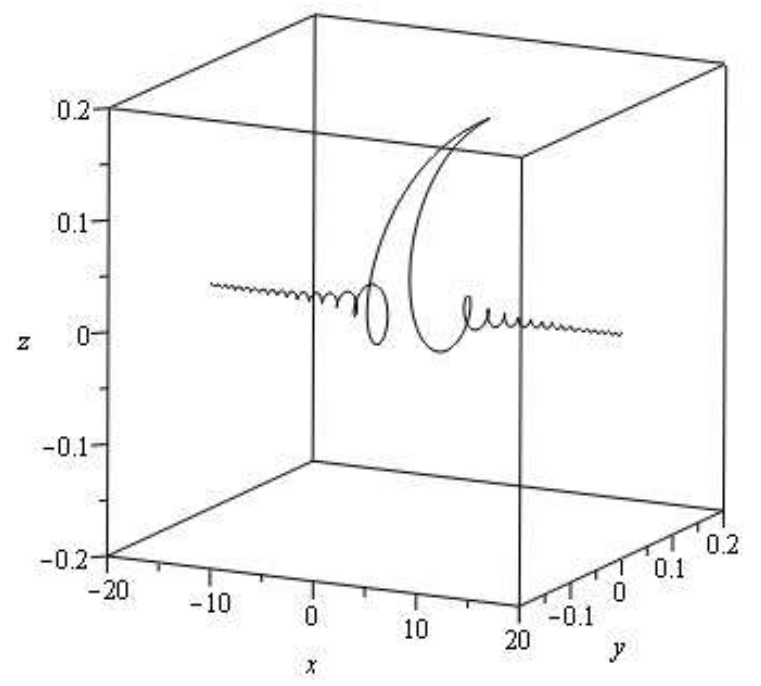

Figure 1: Plot of the similarity solution (60) at $t=1$. This solution takes the form of a disturbance with mavimal curvature near the origin. Farther away from the origin, the oscillations are damped and the filament takes on the form of a line filament, oriented along the $x$-axis. In this plot, we have taken $\epsilon=0.1$.

If we solve equation (51) numerically using (61)-(62) without assuming small amplitude deviations, we can obtain a filament curve with small oscillations along a linear trend with a kink in the center at the origin. This $V$-shaped filament appears as a superposition of the two analytical solutions obtained, above: a linear trend with small amplitude oscillations along that trend line. When the linear trend is removed, we obtain a solution which agrees with the analytical approximation (60) qualitatively. Such solutions are indeed present in the literature in the form of numerical and experimental results. By changing the initial conditions in the numerical simulations, we can obtain a variety of related structures, with various degrees of sharpness in their kink at the origin and various degrees of prevalence of the oscillations along the linear forks. Some of these are shown in Figure 2.

\subsection{Validity of self-similar solutions under the 2D-LIA}

Consider a general similarity solution of the form $\Psi(x, t)=\epsilon \sqrt{2 t} \hat{\Phi}(x / \sqrt{2 t})$ as discussed above. We show that such a solution is always valid under the 2D-LIA provided that $\epsilon$ is small. Recall that $\left|\Psi_{x}\right|=\left|\epsilon \Phi^{\prime}(\eta)+O\left(\epsilon^{3}\right)\right|=\epsilon\left|\Phi^{\prime}(\eta)\right|+O\left(\epsilon^{3}\right)$. Now, $\Phi(\eta)=c_{1} \eta+c_{2} \eta v(\eta)$, so $\Phi^{\prime}(\eta)=c_{1}+c_{2}(v(\eta)+$ $\left.\eta v^{\prime}(\eta)\right)$. Yet, we may show $v(\eta)+\eta v^{\prime}(\eta)=\sqrt{\pi} S(\eta / \sqrt{\pi})-i \sqrt{\pi} C(\eta / \sqrt{\pi})$. Furthermore, observe that $\sqrt{S(\eta / \sqrt{\pi})^{2}+C(\eta / \sqrt{\pi})^{2}}<1$. Therefore, $\left|\Phi^{\prime}(\eta)\right|=\left|c_{1}+\sqrt{\pi} c_{2}\left(S\left(\frac{\eta}{\sqrt{\pi}}\right)-i C\left(\frac{\eta}{\sqrt{\pi}}\right)\right)\right|<$ $\left|c_{1}\right|+\sqrt{\pi}\left|c_{2}\right|$. Now, picking any $c_{1}, c_{2}=O(1)$ in $\epsilon$, we see that $\left|\Psi_{x}\right|=O(\epsilon)$. Since $\epsilon$ was assumed to be small, $\left|\Psi_{x}\right|<<1$, and hence the bounded variation condition for the 2D-LIA holds. As such, the obtained similarity solutions are indeed valid solutions of the 2D-LIA. 


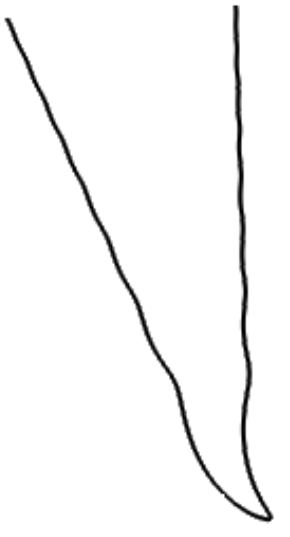

(a)

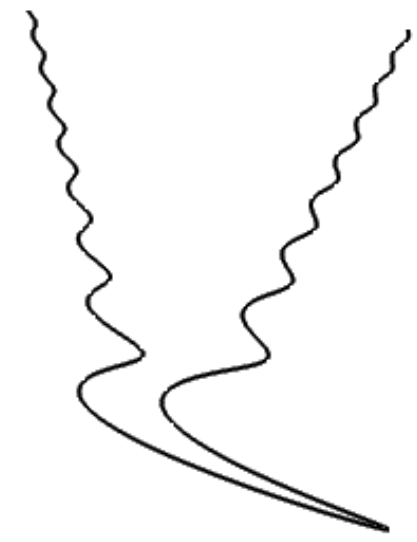

(b)

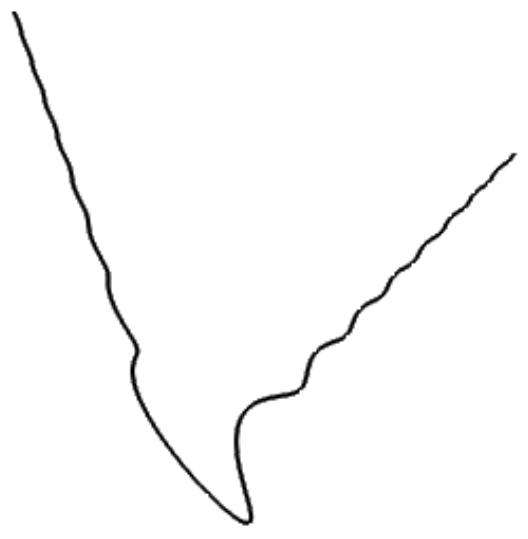

(c)

Figure 2: Plots of example similarity solutions calculated numerically via (61)-(62), at $t=1$. The solutions correspond to various initial conditions as: (a) $p_{0}=0.1, p_{1}=q_{0}=q_{1}=0$, (b) $p_{0}=q_{0}=0.1, p_{1}=q_{1}=0$, (c) $p_{0}=q_{0}=0.1, q_{1}=0.01, p_{1}=0$.

\section{Discussion}

Concerning stationary solutions, both planar and helical filaments exist as two extreme cases, but this has been shown before. In the small amplitude case, more general stationary states are shown to exist in the presence of translation, and we outline the properties of such solutions in the analytic limit where amplitude is small enough. (Otherwise, numerical simulations can be taken if the amplitude is not small enough to permit the analytic limit.) Since we do include translation, large amplitude structures would likely fail to be stable (and hence the solutions would no longer truly be stationary). As such, the types of solutions studied in [28] in the presence of only rotation can exist provided the maximal amplitude of deflection from the reference axis is small enough.

We then considered non-stationary states. A general first integral was obtained, which allowed us to study the wave action preservation properties of the filaments, and a criterion was obtained which ensures wave action conservation. We then considered a number of limits. While we did not assume stationary states, we found that all constant amplitude must result in helical filaments, whereas all solutions with constant phase in space must be planar. Other limits were considered, most interesting the limit where the phase of a solution is large relative to the amplitude of a solution. While the solution in this case is not a true soliton, this limit does yield a solution that exists within a wave envelope with tails that decay rapidly as $x \rightarrow \pm \infty$.

\subsection{Conservation of wave action}

As shown in Section 2, there are a wide variety of possible wave action conserving solutions to the 2D-LIA, in addition to the standard helical and planar vortex filaments. We found that there is a wave action conservation law. We observe that for the planar filament, both of these terms should be zero. This is because of the fact that both terms vanish when the phase is not space dependent. When the two asymptotic limits are equal (due to symmetry) and hence the integral vanishes (as 
in the case of the helix), we also have wave action conservation.

This suggests that purely rotating solutions, such as those discussed in [28], are wave action preserving provided that there is sufficient symmetry. That is, the fact that they are purely rotational does not alone ensure that the solutions are wave action conserving. That said, many of the solutions constructed in [28] do satisfy the wave action conservation condition, as they are symmetric. The planar filaments are a special case of such solutions, and as mentioned above they are always wave action preserving. On the other hand, the helical filaments (modeling Kelvin waves along a vortex filament) are also wave action preserving for any of the contexts in which they arise in this paper, yet this is due to symmetry. Hence, there are multiple ways for solutions to be wave action preserving and satisfy the requirement given above.

It is also clear that solutions such as those that consist of a wave envelope that has decaying amplitude as $x \rightarrow \pm \infty$ will also satisfy the wave action conservation criterion. Therefore, solitary waves and related perturbations along vortex filaments with sufficiently rapidly decaying tails should be wave action conserving.

Finally, we note that the self-similar solutions of Section 4 are not wave action conserving. Under the assumption of a similarity solution $\Psi(x, t)=\sqrt{2 t} \hat{\Phi}(x / \sqrt{2 t})$ as was discussed in Section 5, let us assume that the similarity function $\hat{\Phi}$ is square integrable on the real line, $\int_{-\infty}^{\infty}|\hat{\Phi}(\eta)|^{2} d \eta<\infty$. (For instance, the example solution shown in Figure 1 satisfies this criteria.) Then, our measure of wave action satisfies

$$
E(t)=\int_{-\infty}^{\infty}|\sqrt{2 t} \hat{\Phi}(x / \sqrt{2 t})|^{2} d x=(2 t)^{3 / 2} \int_{-\infty}^{\infty}|\hat{\Phi}(\eta)|^{2} d \eta
$$

Yet, this means $\frac{d E}{d t}>0$ for all $t>0$ (assuming a non-zero solution $\hat{\Phi}$ ). These solutions broaden and unwind as time increases, increasing the square integral of the modulus in the process. This is because the solutions as time $t \rightarrow 0^{+}$have a sharp kink, and the LIA smooths this kink (by broadening the filament profile) at larger timescales at the cost of wave action conservation. Indeed, the Kelvin waves emanating from the kink as it smooths and dissipates is what is causing this increase in wave action over time. In order to have conservation of some kind for such a process, one should consider the non-local dynamics. Then, one would model the total dynamics before, during, and after a reconnection event. As the applicability of the LIA is to the dissipation of the kink after it is already formed, we only capture part of the process, which may explain the lack of conservation we observe.

\subsection{Routes to Kelvin waves in the 2D-LIA}

While helical vortex filaments (corresponding to Kelvin waves) were previously to exist for the 2D-LIA, we have shown that any constant amplitude solution of (6) must result in a helical vortex filament. In the very small amplitude regime, the 2D-LIA reduces to the Schrödinger equation for a free particle, which admits pure plane wave solutions. Other classes of solutions necessarily have non-constant amplitudes, such as the planar or self-similar solutions.

Furthermore, we observe that any solution to (6) which depends on a traveling wave variable must take the form of a shifted helical vortex filament. Physically, this gives Kelvin waves along a line filament (not necessarily centered along the $x$-axis). Therefore, the purely traveling wave solutions of the 2D-LIA must take the form of helical filaments, which greatly retricts the possible traveling wave configurations. However, due to the prevalence of Kelvin waves found along vortex 
filaments in the literature, this might not be particularly surprising. Note that this finding is true when the solution $\Psi$ to (6) depends strictly on a wave variable $z=x-c t$. In the case where the phase of $\Psi$ and the amplitude of $\Psi$ depend on different wave variables (say $z_{1}=x-c_{1} t$ and $z_{2}=x-c_{2} t$ ), this result need not be true. In such a case, one may have a slow amplitude and a fast phase, or vice versa. Since the phase and amplitude would then depend on separate variables, one no longer has a system of compatible ODEs, and another approach would be called for. For this situation, it would prove fruitful to study the 2D-LIA (6) in the context of integrable models. In this context, solutions such as solitons will be possible. The existence of such solutions would make sense, as the 2D-LIA we study here is a variation of the 3D-LIA studied by Hasimoto (with the differences lying in the respective derivations and assumptions invoked), for which solitons and other such solutions have been found. The type of analysis which allows solutions to propagate with different wave speeds in phase and amplitude will be done in another paper, where the integrability of (6) will be exploited in order to obtain solitons and other solutions through a very different approach.

Helical vortex filaments and Kelvin waves are of great interest experimentally. This is motivated by the fact that helical filaments and Kelvin waves are of rather fundamental physical importance, in a few ways. Numerical results and theoretical models have been used to show that a cascade of Kelvin waves transfers energy from large to small length scales [33, 34, 35]. Here, energy is removed from the system via phonon emission [36, 37, 38]. In the classical limit, Kelvin waves have been studied on thin line vortices $[39,40]$ (which correspond to the helical vortex filaments studied here). Kelvin waves have also been studied on knotted vortex rings [41] (which we do not study under the 2D-LIA due to the fact that the formulation assumes vortex filaments aligned along one central axis). Very recently, experimental results for quantum fluids also demonstrated the existence of Kelvin waves along vortex filaments [42]. In fact, it was observed that Kelvin waves are generated by reconnection of quantized vortices, after reconnection events [22] take place. We say more on the post-reconnection events in the next subsection. From this, it is clear that the study of Kelvin waves along vortex filaments is indeed important, and therefore we hope that the various routes to Kelvin wave generation outlined analytically in this paper motivate further work along these lines.

Helical filaments have of course been studied in many contexts in the past, and we know that the LIA results are most useful in the case of small or intermediate wave numbers. In the case of large wave numbers, resulting in a tightly coiled helix, the Biot-Savart integral becomes rapidly varying, hence the non-locality should not be neglected. Hence, for Kelvin waves of a large wave number, one must consider the non-local dynamics governed by the Biot-Savart law [32]. Similar comments hold for the case in which mutual friction effects are taken into account.

\subsection{Self-similar filaments for the 2D-LIA}

While the self-similar solution presented here is approximate, we can numerically integrate the relevant complex ODE in order to recover the true (numerical) solution, and the two were shown to agree. The two solutions agree for the reasonable $O\left(\epsilon^{3}\right)$ solutions we assumed for the approximate solution (60). Note that one can also maintain some of the linear trend in the function $\Phi(\eta)$, which would give oscillations along a line filament that gradually moves away from the $x$-axis. Either way, we observe oscillations along a line filament when we plot the numerical or approximate analytical similarity solutions.

The results are also useful for approximating the vortex kink dynamics in superfluid ${ }^{3} \mathrm{He}$ or su- 
perfluid ${ }^{4} \mathrm{He}[24,25,26]$, since the model (3) is valid in the low-temperature limit (where superfluid friction terms are small and the normal fluid velocity is negligible). From the method employed here, we see that such vortex kinks can essentially be viewed as the union of two straight vortex filament lines on which there are perturbations. These perturbations would be Kelvin waves along the filaments. As time increases, the entire structure smooths. In the case where mutual friction influences are considered $[24,25,26]$, the rate of this smoothing can be enhanced and even dominated due to dissipative effects. However, for the solutions that scale with small variation $\left|\Psi_{x}\right|=O(\epsilon)$, the mutual friction effects for temperatures less than $2 \mathrm{~K}$ can be viewed as even smaller perturbations, of order $O\left(\epsilon^{2}\right)$ or higher. Therefore, the similarity solutions here demonstate the desired qualitative properties, although for quantitatively accurate approximations, one should use the quantum form of the LIA as given by [4]. This was considered in [24, 25, 26] for the quantum LIA with mutual friction and normal fluid effects, and the results here agree qualitatively with those studies.

Furthermore, and more importantly, we see agreement between the self-similar solutions and both numerical and experimental results in the literature for superfluid vortex filament dynamics. Physically, the vortex kink solutions correspond to the vortex filament structures previously studied in $[43,42]$ following reconnection events. In particular, two vortex filaments were permitted to cross, with a reconnection event occurring at some fixed time. Before this time, the filaments were two distinct line filaments, whereas after the reconnection there were two distinct V-shaped vortex filaments (corresponding to vortex kinks); see Figure 1 of reference [42] for a schematic of this process. It is these post-reconnection events that are nicely modeled by our mathematical formulation. It was shown experimentally in [42] that Kelvin waves propagate along such V-shaped filaments (these correspond to the wavy structure seen in the small-time plots of Figure 2 in the present paper). What we see from the present results is that for small time values (immediately after such a reconnection when the new V-shaped filaments are created) the Kelvin waves along the filament are at their largest, while for increasing values of time there is a smoothing effect (the excitation due to the reconnection event dies off) and the filament structure essentially tends toward a smoother $U$-shaped curve. Therefore, the mathematical formulation of Section 4 can allow one to study post-reconnection events of the type experimentally confirmed in [42].

The vortex reconnection event described experimentally in [42] was considered theoretically in [22]. In [22], it was shown that the post-intersection dynamics can involve the creation of vortex rings for small time, before the two predominant V-shaped filaments dominate. During this time, the V-shaped filaments exhibit the largest amplitude Kelvin waves. As time progresses, there is a smoothing effect and the waves along the filament gradually dissipate leaving a more uniform structure. Again, this is exactly the type of behavior observed in the matched self-similar solutions constructed in this section. This behavior is shown for the special case depicted in Figure 2. In [44], the relation between kinks and waves emanating from said kinks was discussed in the context of models which preserve vortex filament cores. Kinks are shown to form after reconnection events, and are directly responsible for certain waves which then propagate along the vortex filament. Again, this is essentially what we see in our solutions: at small times, the waves are most pronounced near the origin (the location of the kink). For larger times, these waves propagate away from the kink and the overall structure smooths. Note that the specific experimental results of [42] actually supports the similarity assumption we use in constructing our solutions. That is to say, the manner of propagation of the Kelvin waves observed in [42] was shown to correspond to what one expects from a theoretical assumption of self-similarity. Now, while our results show qualitative agreement with these studies, quantitative agreement would require the inclusion of mutual friction effects, as 
mentioned above. Further, to deal with sharp kinks at reconnection events, as well as tightly coiled Kelvin waves, one would want to consider the full Biot-Savart dynamics of the vortex filaments. Demonstrating similarity solutions has not been done for the full non-local Biot-Savart dynamics, and this will be the topic of a future work.

\subsection{Boundary conditions}

While the solutions here are define over an unbounded domain (they are perturbations of a line filament over the real line which can move in all of $\mathbb{R}^{3}$ ), it is possible to consider the extension of such solutions to bounded domains. A type of periodicity condition can be used in the case of helical or planar filaments. Such conditions would then give eigenvalue problems, in effect locking the wave number or spectral parameter to a discrete spectrum. In general, these parameters exist on a continuum. In the helical case, we take the wave number $k>0$, while in the planar case we take a spectral parameter $\omega<0$. If we give periodic boundary conditions at solid boundaries then the discrete subset of values can be obtained. In the case of a line filament, a condition at the solid boundary may be that the line remains stationary. Now, the 'tails' of the similarity solutions take on the form of line filaments as one moves further away from the origin, hence one can expect that asymptotically these tails will behave like line filaments and therefore that boundary conditions on line filaments can be used for these tails. The exception to this is if the bounding domain is too small, in which case the core region of these solutions could be distorted by boundary effects.

\section{References}

[1] Hall, H.E. \& Vinen, W.F. 1956 The rotation of liquid helium II. I. Experiments on the propagation of second sound in uniformly rotating helium II, Proc. R. Soc. London A 238, 204.

[2] Hall, H.E. \& Vinen, W.F. 1956 The rotation of liquid helium II. II. The theory of mutual friction in uniformly rotating helium II, Proc. R. Soc. London A 238, 215.

[3] Bekarevich, I.L. \& Khalatnikov, I.M. 1961 Phenomenological derivation of the equations of vortex motion in He II, Sov. Phys. JETP 13, 643.

[4] Schwarz, K.W. 1985 Three-dimensional vortex dynamics in superfluid ${ }^{4}$ He: Line-line and lineboundary interactions, Phys. Rev. B 31, 5782.

[5] Arms, R.J. \& Hama, F.R. 1965 Localized-induction concept on a curved vortex and motion of an elliptic vortex ring. Phys. Fluids 8, 553.

[6] Da Rios, L.S. 1906 Sul moto d'un liquido indefinite con un filetto vorticoso di forma qualunque. Rend. Circ. Mat. Palermo 22, 117.

[7] Hasimoto, H. 1972 A soliton on a vortex filament, J. Fluid Mech. 51, 477.

[8] Kida, S. 1981 A vortex filament moving without change of form. J. Fluid Mech. 112, 397.

[9] Hasimoto, H. 1971 Motion of a Vortex Filament and its relation to Elastica, Journal of the Physical Society of Japan 31, 293. 
[10] Kida, S. 1982 Stability of a steady vortex filament, Journal of the Physical Society of Japan $51,1655$.

[11] Fukumoto, Y. 1997 Stationary configurations of a vortex filament in background flows, Proceedings of the Royal Society of London A 453, 1205.

[12] Umeki, M. 2010 A locally induced homoclinic motion of a vortex filament. Theor. Comput. Fluid Dyn. 24, 383.

[13] Umeki, M. 2013 A real-space representation of a locally induced vortex filament, Theor. Appl. Mech. Jpn. 61, 195.

[14] Van Gorder, R.A. 2012 Exact solution for the self-induced motion of a vortex filament in the arclength representation of the local induction approximation, Phys. Rev. E 86, 057301.

[15] Van Gorder, R.A. 2013 Scaling laws and accurate small-amplitude stationary solution for the motion of a planar vortex filament in the Cartesian form of the local induction approximation, Phys. Rev. E 87, 043203.

[16] Svistunov, B. 1995 Superfluid turbulence in the low-temperature limit, Phys. Rev. B 52, 3647.

[17] Boffetta, G., Celani, A., Dezzani, D., Laurie, J., \& Nazarenko, S. 2009 Modeling Kelvin Wave Cascades in Superfluid Helium, J. Low Temp. Phys. 156, 193.

[18] Sonin, E.B. 2012 Symmetry of Kelvin-wave dynamics and the Kelvin-wave cascade in the T=0 superfluid turbulence, Phys. Rev. B, 85, 104516.

[19] Sonin, E.B. 2012 Dynamics of helical vortices and helical-vortex rings, EPL 97, 46002.

[20] Barenghi, C. F. \& Donnelly, R. J. 2009 Vortex rings in classical and quantum systems. Fluid Dynamics Research, 41(5), 051401.

[21] Ricca, R. L., Samuels, D. C., \& Barenghi, C. F. 1999 Evolution of vortex knots. Journal of Fluid Mechanics 391, 29.

[22] Kursa, M., Bajer, K., \& Lipniacki, T. 2011 Cascade of vortex loops initiated by a single reconnection of quantum vortices. Physical Review B 83(1), 014515.

[23] Kleckner, D., \& Irvine, W. T. 2013 Creation and dynamics of knotted vortices. Nature Physics $9(4), 253$.

[24] Lipniacki, T. 2003 Shape-preserving solutions for quantum vortex motion under localized induction approximation, Physics of Fluids 15, 1381.

[25] Lipniacki, T. 2003 Quasi-static solutions for quantum vortex motion under the localized induction approximation. J. Fluid Mech. 477, 321.

[26] Van Gorder, R. A. 2013 Self-similar vortex dynamics in superfluid ${ }^{4}$ He under the Cartesian representation of the Hall-Vinen model including superfluid friction. Phys. Fluids 25, 095105.

[27] Van Gorder, R.A. 2014 Quantum vortex dynamics under the tangent representation of the local induction approximation. Journal of Fluid Mechanics 740, 5. 
[28] Van Gorder, R.A. 2014 General rotating quantum vortex filaments in the low-temperature Svistunov model of the local induction approximation. Phys. Fluids 26, 065105.

[29] Hietala, N. \& Hänninen, R. 2014 Comment on "General rotating quantum vortex filaments in the low-temperature Svistunov model of the local induction approximation" [Phys. Fluids 26, 065105 (2014)]." Phys. of Fluids 26, 119101.

[30] Van Gorder, R. A. 2014 Response to "Comment on 'General rotating quantum vortex filaments in the low-temperature Svistunov model of the local induction approximation"” [Phys. Fluids26, 119101 (2014)]." Phys. Fluids 26, 119102.

[31] Van Gorder, R. A. 2013 Motion of a helical vortex filament in superfluid 4He under the extrinsic form of the local induction approximation, Phys. Fluids 25, 085101.

[32] Van Gorder, R.A. 2015 Helical vortex filament motion under the non-local Biot-Savart model. Journal of Fluid Mechanics 762, 141.

[33] Kivotides, D., Vassilicos, J.C., Samuels, D.C., \& Barenghi, C.F. (2001) Kelvin waves cascade in superfluid turbulence. Phys Rev Lett 86(14), 3080-3083.

[34] Lvov, V., Nazarenko, S., \& Rudenko, O. (2007) Bottleneck crossover between classical and quantum superfluid turbulence. Phys Rev B 76(2), 024520.

[35] Kozik, E.V. \& Svistunov B.V. (2009) Theory of decay of superfluid turbulence in the lowtemperature limit. J Low Temp Phys 156, 215-267.

[36] Nore, C., Abid, M., \& Brachet, M. (1997) Kolmogorov turbulence in low-temperature superflows. Phys Rev Lett 78, 3896-3899.

[37] Leadbeater, M., Winiecki, T., Samuels, D.C., Barenghi, C.F., \& Adams, C.S. (2001) Sound emission due to superfluid vortex reconnections. Phys Rev Lett 86(8), 1410-1413.

[38] Vinen, W.F., Tsubota, M., \& Mitani, A. (2003) Kelvin-wave cascade on a vortex in superfluid $4 \mathrm{He}$ at a very low temperature. Phys Rev Lett 91(13), 135301.

[39] Hopfinger, E.J. \& Browand, F.K. (1982) Vortex solitary waves in a rotating, turbulent flow. Nature 295, 393-395.

[40] Maxworthy, T., Hopfinger, E.J., \& Redekopp, L.G. (1985) Wave motions on vortex cores. J Fluid Mech 151, 141-165.

[41] Kleckner, D. \& Irvine, W.T.M. (2013) Creation and dynamics of knotted vortices. Nature Phys 9, 253-258.

[42] Fonda, E., Meichle, D.P., Ouellette, N.T., Hormoz, S., and Lathrop, D.P. 2014 Direct observation of Kelvin waves excited by quantized vortex reconnection. Proc. Natl. Acad. Sci. USA $111,4707$.

[43] Fonda, E., Meichle, D.P., Ouellette, N.T., Hormoz, S., Sreenivasan, K.R., and Lathrop, D.P. 2012 Visualization of Kelvin waves on quantum vortices. arXiv:1210.5194. 
[44] Kerr, R.M. (2011) Vortex Stretching as a Mechanism for Quantum Kinetic Energy Decay. Phys. Rev. Lett. 106, 224501. 